\title{
Dilute Solution Properties of Monodisperse Poly( $\alpha$-Methylstyrene) \\ II. Hydrodynamic Properties in Good Solvents
}

\author{
Kuniaki SaKato and Michio Kurata \\ Institute for Chemical Research, Kyoto University, Uji, Kyoto, Japan.
}

(Received December 20, 1969)

\begin{abstract}
Hydrodynamic properties of monodisperse poly( $\alpha$-methylstyrene) were studied in dilute benzene solutions, both concentration and molecular weight being varied. Molecular weight determination was performed by three methods, osmometry, light scattering and sedimentation equilibrium. The intrinsic viscosity was found to be $[\eta]=\left(1.03_{4} \pm\right.$ $0.05) \times 10^{-4} M^{0.72} \mathrm{~d} l / \mathrm{g}$ at $30^{\circ} \mathrm{C}$, where $M$ is the molecular weight. The sedimentation coefficient extrapolated to zero concentration and one atmosphere was found to be $s_{0}{ }^{\circ}=(3.61 \pm$ $0.03) \times 10^{-15} M^{0.43} \mathrm{sec}$. The Scheraga-Mandelkern constant $\beta$ was evaluated as $(2.43 \pm 0.03) \times$ $10^{6}$ which was independent of $M$ and in agreement with the value previously obtained in theta solvent. The concentration dependence of sedimentation coefficient was found to be compatible with the recent theory by Pyun and Fixman.
\end{abstract}

KEY WORDS Monodisperse / Poly $(\alpha$-Methylstyrene) / Intrinsic Viscosity / Sedimentation / Scheraga-Mandelkern Constant / Molecular Weight / Second Virial Coefficient / Excluded Volume / Draining / Pyun-Fixman Theory /

Based on extensive measurements of intrinsic viscosity of anionically prepared polystyrene, Berry ${ }^{1}$ has concluded that polymer chains in good sovlent exhibit a marked effect of partial draining even when the molecular weight $M$ is as high as $5 \times 10^{5}-8 \times 10^{5}$. However, this conclusion seems to be not necessarily definitive, ${ }^{2-4}$ because the analysis of data is based on the assumption that the thermodynamic interaction parameter of chain segments is a linear function of $1 / T$ over a wide range of temperature $T$.

The most direct method of studying the hydrodynamic draining effect should consist of measurements of the intrinsic viscosity $[\eta]$ and the mean square radius of gyration $\left\langle S^{2}\right\rangle$ of monodisperse polymers as a function of polymer molecular weight $M$. Such measurements enable us to evaluate the so-called "Flory constant" $\Phi$ by the relation

$$
[\eta]=6^{3 / 2} \Phi\left\langle S^{2}\right\rangle^{3 / 2} / M
$$

free from the uncertainty concerning the heterogeneity correction. According to the present theories, the quantity $\Phi$ is not a constant but an increasing function of $M$ if the draining effect exists. $^{5-8}$ In the nondraining limit corresponding to sufficiently large $M$, it attains the asymptotic value, $c a$. $2.6 \times 10^{21}$. Thus, observation of the behavior of $\Phi$ as function of $M$ is expected to reveal the nature of the hydrodynamic draining of polymer coil. However, this line of approach is often confronted with the familiar difficulty in precise determination of $\left\langle S^{2}\right\rangle$ by light scattering measurements, especially at low and high molecular weight sides. Furthermore, the quantity $\Phi$ is appreciably affected by the excluded volume effect, ${ }^{6,9}$ and this complicates the analysis of the problem. Thus, in this series of study, another method, though less direct than the above, is attempted to study the draining effect, which consists of measurements of the intrinsic viscosity and the sedimentation coefficient.

In a preceding paper, ${ }^{10}$ we presented the results of viscosity and sedimentation velocity measurements on anionically-prepared poly $(\alpha$-methylstyrene) in theta solvent, cyclohexane at $39^{\circ} \mathrm{C}^{*}$.

* The theta temperature in cyclohexane was $38.6^{\circ} \mathrm{C}$ for sample $\mathrm{A}-1$ and $37.8^{\circ} \mathrm{C}$ for sample $\mathrm{BH}-1$. Both samples were anionically prepared in tetrahydrofuran by using sodium-naphthalene and $n$-butyl lithium as initiator, respectively. The latter is in close agreement with the value $37.0^{\circ} \mathrm{C}$ reported by Cowie and Bywater (J. Polym. Sci., Part A-2, 6, 499 (1968)) for the same type of polymers as our BH and $\mathrm{BB}$ series, but is slightly higher than the value $35.0^{\circ} \mathrm{C}$ by Kato et al. (J. Phys. Chem., 72, 2161 (1968)). Thus, the used condition $39.0^{\circ} \mathrm{C}$ may not correspond exactly to the theta point especially for samples of the $\mathrm{BH}$ and $\mathrm{BB}$ series, but is very close to it ( $c f$. statements given in the paragraph following Eq. 4). 
The Scheraga-Mandelkern constant $\beta$ was found to be $2.41 \times 10^{6}$ which was considerably lower than the theoretical value for the nondraining Gaussian chains, ca. $2.7 \times 10^{6}$. However, it was not able to be determined whether the discrepancy was attributed to a real physical effect such as the draining effect or simply to errors possibly involved in our molecular weight determination by light scattering. Thus, the need for a re-examination of the molecular weight values has arisen in order that the question be resolved. This paper intends to meet the need by making sedimentation equilibrium experiments. Viscosity and sedimentation velocity measurements are also performed in good solvent, benzene at $30^{\circ} \mathrm{C}$, and comparison of the results with those obtained in theta solvent is made in the hope of obtaining information concerning the draining effect and the excluded volume effect.

\section{EXPERIMENTAL}

\section{Polymer Samples and Solvents}

The nine samples of $\operatorname{poly}(\alpha$-methylstyrene $)$ with narrow molecular weight distributions which were prepared for viscosity and sedimentation velocity measurements in Part I were chosen for the present study. Cyclohexane was the same as that used in Part I. Benzene was put in physical measurements after being dried with sodium metal and fractionally distilled.

\section{Light Scattering}

The Brice type photoelectric photometer (type PG-21, Shimadzu Seisakusho, Ltd., Kyoto) was used for light scattering measurements. The cell assembly consisted of a cylindrical cell and its surrounding jacket which was thermostated by circulating water. All measurements were made on the vertical component of scattered light for the vertically-polarized incident beam of $436 \mathrm{~m} \mu$ wavelength. The photometer used was calibrated to the Rayleigh ratio of pure benzene by using the Carr and Zimm value, $R_{90}=49.5 \times 10^{-6}$ at $30^{\circ} \mathrm{C}^{11}$ The refractive index increments $d n / d c$ were determined with a Debye type differential refractometer which was calibrated with 1.087 wt $\% \mathrm{KCl}$ aqueous solution at $25^{\circ} \mathrm{C}^{12}$ The results for $436 \mathrm{~m} \mu$ were $0.130_{4} \mathrm{ml} / \mathrm{g}$ in benzene at $30^{\circ} \mathrm{C}$ and $0.200 \mathrm{ml} / \mathrm{g}$ in cyclohexane at $39^{\circ} \mathrm{C}$, respectively.
Sample solutions were clarified by spinning for about one hour at $20000 \mathrm{rpm}$ in a preparative centrifuge equipped with a temperature control unit (type 55P, Hitachi Ltd., Tokyo), and then the solutions were pipetted directly into the light scattering cell. Measurements of the scattered light intensity were carried out on at least four concentrations in the range of $0.04-1.0 \mathrm{wt} \%$ and at thirteen scattering angles between $30^{\circ}$ and $150^{\circ}$.

\section{Sedimentation Equilibrium}

Sedimentation equilibrium measurements were carried out according to the procedure established by Utiyama et al. ${ }^{13}$ in a Spinco Model E ultracentrifuge equipped with an RTIC temperature control unit. We used the Rayleigh interferometer as an optical system for detecting the polymer concentration gradient. The fringe shifts per unit polymer concentration were $0.5292 \mathrm{~cm} \cdot \mathrm{d} l / \mathrm{g}$ in cyclohexane at $39^{\circ} \mathrm{C}$ and 0.0338 $\mathrm{cm} \cdot \mathrm{d} l / \mathrm{g}$ in benzene at $30^{\circ} \mathrm{C}$, respectively, for the light of $546 \mathrm{~m} \mu$ wavelength. A multicell rotor (An-G type) with laterally-wedged double sector cells was used. Measurements were performed in the range of concentration of $0.1-0.5$ wt $\%$ without using bottom liquid. The column height of the solution was $0.15 \mathrm{~cm}$. In the case of measurements on higher molecular weight samples, the time necessary to attain the equilibrium state was reduced with the aid of the overspeed technique of Hexner and others. ${ }^{14}$

\section{Sedimentation Velocity}

Method and procedures for obtaining the sedimentation coefficient at zero concentration and one atmosphere were the same as those described in detail in Part I of this series. ${ }^{10}$ Measurements were made in benzene at $30^{\circ} \mathrm{C}$ and at rotor speed $42040 \mathrm{rpm}$. The partial specific volume of polymer at infinite dilution $\bar{v}^{\circ}$ and the solvent density $\rho_{0}$ were determined as $\bar{v}^{\circ}=0.883$ $\mathrm{m} l / \mathrm{g}$ and $\rho_{0}=0.867 \mathrm{~g} / \mathrm{m} l$, respectively, in benzene at $30^{\circ} \mathrm{C}$ by using a measuring-cylindrical pycnometer.

\section{Osmotic Pressure}

Osmotic pressure measurements were also performed for all samples (BB-5 and BB-7 excepted) in toluene at $37^{\circ} \mathrm{C}$ by using a Mechrolab high speed membrane osmometer fitted with ultracellafilter of grade "allerfeinst" as a semi- 
permeable membrane.

\section{Intrinsic Viscosity}

Dilution viscometers of the Ubbelohde type were used for viscosity measurements. The kinetic energy correction and the shear dependence of solution viscosity were both negligible under the experimental conditions. The solvent viscosity of benzene was 0.566 centi-poise at $30^{\circ} \mathrm{C}$.

\section{RESULTS}

\section{Molecular Weight and the Second Virial Coefficient from Light Scattering Measurements}

Analyses of the light scattering data were made by the familiar procedure of Zimm, taking $\left(K_{v} c\right)$ $\left.R_{\theta}\right)^{1 / 2}$, instead of $K_{v} c / R_{\theta}$, as the ordinate value. ${ }^{15,16}$ Here $c$ is the polymer concentration in $\mathrm{g} / \mathrm{m} l, R_{\theta}$ is the reduced intensity of scattered light at angle $\theta$ and $K_{v}$ is the light scattering factor for the vertically polarized light. The weight-average molecular weight $M_{w}$, the second virial coefficient $A_{2}$ and the $z$-average value of the mean-square radius of gyration $\left\langle S^{2}\right\rangle_{z}$ were evaluated as usual. The results are shown in Table I. We omitted values of $\left\langle S^{2}\right\rangle_{z}$ from the table because of their poor reliability. In order to obtain accurate estimates of $\left\langle S^{2}\right\rangle_{z}$, it is necessary to conduct light scattering measurements at lower angles than $30^{\circ}$. Such measurements are now in progress in our institute by using a low-angle light-scattering photometer ${ }^{16}$ and the results will be published elsewhere.

\section{Molecular Weight from Osmotic Pressure}

The number average molecular weight $M_{n}$ was evaluated from osmotic pressure data by using the familiar $(\pi / c R T)^{1 / 2}$ vs. $c$ plot. Here $\pi$ is the osmotic pressure and $R T$ has the usual meaning. Table I includes the number-average molecular weights thus obtained, of which the values for the samples $\mathrm{BB}-8$ and $\mathrm{BH}-1$ are based on new measurements. ${ }^{10}$

\section{Molecular Weight and the Second Virial Coefficient from Sedimentation Equilibrium Measurements}

The weight average molecular weight $M_{w}$ and the light scattering second virial coefficient $A_{2}$ are also obtainable from sedimentation equilibrium experiments. According to the theory of sedimentation equilibrium in the binary system of monodisperse polymer and solvent, the following
Table I. Molecular weight and light-scattering second virial coefficient

\begin{tabular}{lccccc}
\hline \multirow{2}{*}{$\begin{array}{c}\text { Polyme } \\
\text { code }\end{array}$} & \multicolumn{2}{c}{$M_{w} \times 10^{-4}$} & $M_{n} \times 10^{-4}$ & \multicolumn{2}{c}{$A_{2} \times 10^{4}$} \\
\cline { 2 - 5 } & $\mathrm{LS}^{\mathrm{a}}$ & $\mathrm{SE}^{\mathrm{a}}$ & & LS $^{\mathrm{c}}$ & $\mathrm{SE}^{\mathrm{c}}$ \\
\hline $\mathrm{BB}-8$ & $4.03^{\mathrm{d}}$ & $4.84^{\mathrm{c}}$ & 4.24 & $\ldots$ & 6.50 \\
$\mathrm{BB}-4$ & $4.87^{\mathrm{b}}$ & $5.60^{\mathrm{d}}$ & 5.26 & 6.01 & $\ldots$ \\
$\mathrm{BH}-1$ & $26.0^{\mathrm{b}}$ & $26.1^{\mathrm{b}}$ & 26.0 & 3.89 & 3.60 \\
$\mathrm{BB}-9$ & $35.5^{\mathrm{c}}$ & $37.4^{\mathrm{b}}$ & 35.8 & 3.82 & 3.50 \\
$\mathrm{BB}-6$ & $48.8^{\mathrm{b}}$ & $41.6^{\mathrm{d}}$ & 48.7 & 3.31 & $\ldots$ \\
$\mathrm{BB}-10$ & $64.0^{\mathrm{b}}$ & $69.0^{\mathrm{b}}$ & 69.0 & 3.21 & 3.20 \\
$\mathrm{~A}-1$ & $97.0^{\mathrm{b}}$ & $\ldots$ & 93.2 & 3.59 & $\ldots$ \\
$\mathrm{BB}-5$ & $143^{\mathrm{b}}$ & $143^{\mathrm{b}}$ & $\ldots$ & 2.26 & 2.69 \\
$\mathrm{BB}-7$ & $168^{\mathrm{b}}$ & $167^{\mathrm{b}}$ & $\ldots$ & 2.10 & 2.60 \\
\hline
\end{tabular}

a LS: Light-scattering, SE: Sedimentation equilibrium.

b Determined in both benzene and cyclohexane.

c Determined in benzene at $30^{\circ} \mathrm{C}$.

d Determined in cyclohexane at $39^{\circ} \mathrm{C}$.

expression is obtained for the polymer concentration at meniscus $c\left(r_{a}\right)$ and that at cell bottom $c\left(r_{b}\right)::^{17}$

$$
\begin{gathered}
M_{a p p}=M^{-1}+A_{2}\left[c\left(r_{a}\right)+c\left(r_{b}\right)\right] \\
+A_{3}^{*} M\left\{\left[c\left(r_{a}\right)\right]^{2}+c\left(r_{a}\right) c\left(r_{b}\right)+\left[c\left(r_{b}\right)\right]^{2}\right\}+\ldots
\end{gathered}
$$

with

$$
M_{a p p}=\left[c\left(r_{b}\right)-c\left(r_{a}\right)\right] / c_{0} \Lambda
$$

and

$$
\Lambda=\left(1-\bar{v}^{\circ} \rho_{0}\right) \omega^{2}\left(r_{b}{ }^{2}-r_{a}{ }^{2}\right) / 2 R T
$$

Here $c_{0}$ is the initial concentration of the test solution, $A_{3}{ }^{*}$ is a constant closely related to the third virial coefficient and $\omega$ is the angular speed of the rotor. Figure 1 shows the plot of $M_{a p p}{ }^{-1}$ against $\left[c\left(r_{a}\right)+c\left(r_{b}\right)\right] / 2$ for the data in benzene at $30^{\circ} \mathrm{C}$ and closed circles represent the data obtained in cyclohexane at $39^{\circ} \mathrm{C}$. The circles show a non-zero (positive) slope in the plot, indicating that the given temperature is slightly higher than the theta temperature of this sample. In the figure, the abscissa $\left[c\left(r_{a}\right)+c\left(r_{b}\right)\right] / 2$ is chosen instead of $\left[c\left(r_{a}\right)+c\left(r_{b}\right)\right]$.

The sedimentation equilibrium data can also be analyzed by using the theoretical expression derived for the multicomponent system of polydisperse polymers. In this case, $M_{a p p}$ defined by Eq. 3 can be expressed in the form ${ }^{17}$

$$
M_{a p p}{ }^{-1}=M_{w}{ }^{-1}+2 A_{2} G(\Lambda) c_{0}+O\left(c_{0}{ }^{2}\right)
$$


Dilute Solution Properties of Monodisperse_Poly $(\alpha$-Methylstyrene)

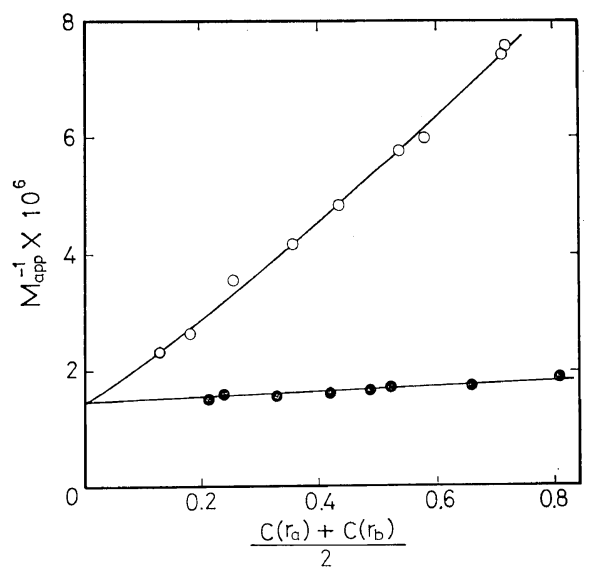

Figure 1. Analysis of the sedimentation equilibrium data according to Eq. 2. Sample: BB-10. Solvents: Benzene at $30^{\circ} \mathrm{C}$ (white circles); Cyclohexane at $39^{\circ} \mathrm{C}$ (black circles).

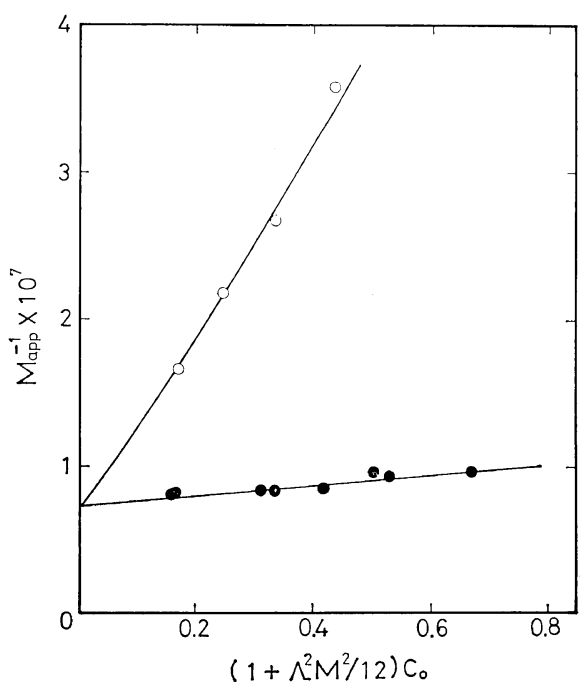

Figure 2. Analysis of the sedimentation equilibrium data according to Eq. 5. Sample: BB-5. Solvents: Benzene at $30^{\circ} \mathrm{C}$ (white circles); Cyclohexane at $39^{\circ} \mathrm{C}$ (black circles).

with

$$
\begin{aligned}
& G(\Lambda)=1+\left[\left(\Lambda M_{z}\right)^{2} / 12\right]+\left[\left(\Lambda M_{z}\right)^{4} / 720\right] \\
& \times\left[\left(M_{z+1} / M_{z}\right)^{2}-2\left(M_{z+1}{ }^{2} M_{z+2}{ }^{2} / M_{z}^{4}\right)\right]+\ldots
\end{aligned}
$$

Here $M_{z}, M_{z+1}$ and $M_{z+2}$ represent $z$-average, $(z+1)$-average and $(z+2)$-average values of the polymer molecular weight in the original

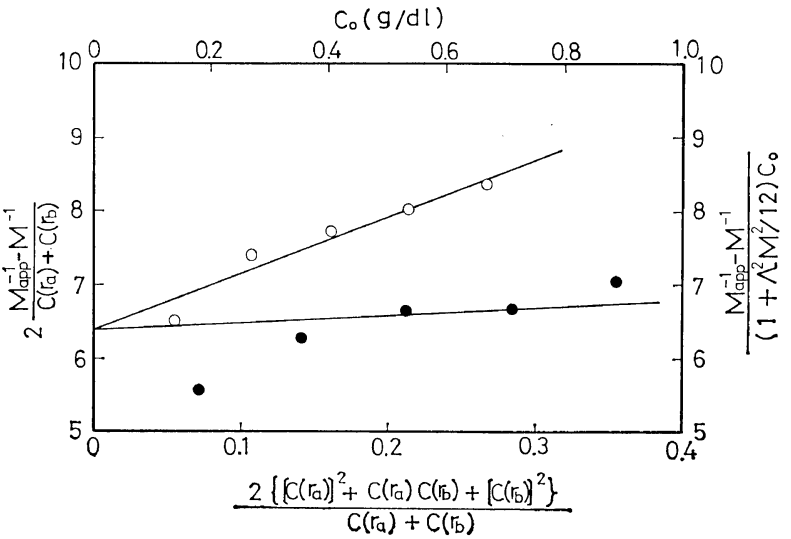

Figure 3. Determination of the second virial coefficient $A_{2}$ from sedimentation equilibrium. Sample: BB-10. Solvent: Benzene at $30^{\circ} \mathrm{C}$.

solution, respectively. Under the experimental condition such that $\Lambda M_{z} \simeq 1$ and $M_{z} / M_{w} \simeq$ $M_{z+1} / M_{z} \simeq M_{z+2} / M_{z+1} \simeq 1$, the terms higher than $\left(\Lambda M_{z}\right)^{2}$ can be ignored. Hence,

$$
G(\Lambda)=1+\left[\left(\Lambda M_{z}\right)^{2} / 12\right]
$$

Figure 2 illustrates the plot of $M_{a p p}{ }^{-1}$ against $\left[1+(\Lambda M)^{2} / 12\right] c_{0}$ where closed circles represent the data obtained for sample BB-5 in cyclohexane and open circles the data in benzene. The molecular weight $M_{w}$ obtained by this type of plot is in good agreement with the value of $M$ obtained by the plot of the type of Figure 1. The results are shown in Table I.

Evaluation of the second virial coefflcient $A_{2}$ for benzene solutions is subjected to larger errors because of the concave upward curvature of the plots in Figures 1 and 2. Thus, for evaluation of $A_{2}$, we replotted the data according to the following equations:

$$
\begin{aligned}
& \frac{2\left(M_{a p p}{ }^{-1}-M^{-1}\right)}{c\left(r_{a}\right)+c\left(r_{b}\right)}=2 A_{2} \\
& +2 A_{3}{ }^{*} M \frac{\left[c\left(r_{a}\right)\right]^{2}+c\left(r_{a}\right) c\left(r_{b}\right)+\left[c\left(r_{b}\right)\right]^{2}}{c\left(r_{a}\right)+c\left(r_{b}\right)}
\end{aligned}
$$

and

$$
\frac{M_{a p p}{ }^{-1}-M_{w}{ }^{-1}}{\left[1+\left(\Lambda M_{z}\right)^{2} / 12\right] c_{0}}=2 A_{2}+\mathrm{O}\left(c_{0}\right)
$$

Figure 3 shows the plots based on these equations. 
In spite of a rather large scattering of the plots which is unavoidable especially at high dilutions, it is possible to draw two straight lines with a common intercept at the ordinate. The second virial coefficient $A_{2}$ can be evaluated from the intercept. The results are also given in Table I, which are favorably compared with the values directly obtained from light scattering data.

\section{Sedimentation Coefficient and its Concentration} Dependence Coefficient

The sedimentation coefficient at infinite dilution and one atmosphere $s_{0}{ }^{\circ}$ was determined by using the Fujita theory of the pressure effect and the best-fit method for zero-time correction. ${ }^{10}$ The results are shown in Table II where the values of the concentration dependence coefficient $k_{s}$ are also tabulated. The double logarithmic plots of $s_{0}^{\circ}$ against $M_{w}$ are shown by open circles in Figure 4. Since two values of $M_{w}$ are available for each sample of BB-8, BB-9 and BB-6 as shown in Table $I$, we give two circles for each of these polymers. The data obtained in benzene at $30^{\circ} \mathrm{C}$ are best represented by

$$
s_{0}{ }^{\circ}=(3.61 \pm 0.03) \times 10^{-15} M^{0.43} \mathrm{sec}
$$

Here we omitted the subscript of $M$, for the monodispersity of these samples has already been confirmed within experimental error. In the figure, the data obtained in cyclohexane at $39^{\circ} \mathrm{C}$ are also shown by closed circles and by circles with vertical bars, the former representing our data and the latter those of Noda et al. ${ }^{18}$ The straight line represents the relationship,

$$
\left(s_{0}^{\circ}\right)_{\theta}=(2.00 \pm 0.03) \times 10^{-15} M^{0.50} \mathrm{sec}
$$

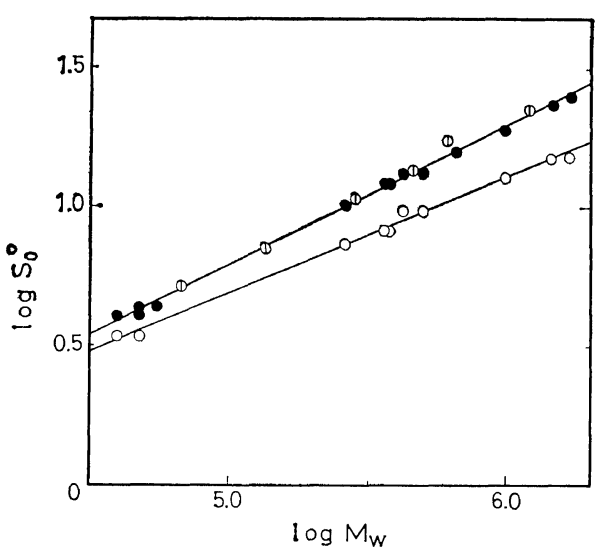

Figure 4. Double logarithmic plots of $s_{0}^{\circ}$ against $M_{w}$. Solvents: Benzene at $30^{\circ} \mathrm{C}$ (white circles); Cyclohexane at $39^{\circ} \mathrm{C}$ (black circles, present data; circles with vertical bars, data by Noda et al.)

\section{Intrinsic Viscosity}

The intrinsic viscosity [ $\eta]$ and the Huggins coefficient $k^{\prime}$ were determined by using both the $\eta_{s p} / c v s . c$ plot and the $\ln \eta_{r e l} / c$ vs. $c$ plot. The results are summarized in Table II. Figure 5 shows the double logarithmic plot of $[\eta]$ against $M_{w}$. The open circles represent the data obtained in benzene at $30^{\circ} \mathrm{C}$, which are well represented by

$$
[\eta]=\left(1.03_{4} \pm 0.05\right) \times 10^{-4} M^{0.72} \mathrm{~d} l / \mathrm{g}
$$

The closed circles represent our theta-point data given in Table II, and the circles with vertical bars represent the data obtained by Noda et $a l .^{18}$ The former are best represented by

$$
[\eta]_{\theta}=(7.60 \pm 0.07) \times 10^{-4} M^{0.50} \mathrm{~d} l / g
$$

\begin{tabular}{|c|c|c|c|c|c|c|c|c|}
\hline \multirow{2}{*}{$\begin{array}{l}\text { Polymer } \\
\text { code }\end{array}$} & \multicolumn{4}{|c|}{ Benzene $\left(30^{\circ} \mathrm{C}\right)$} & \multicolumn{4}{|c|}{ Cyclohexane $\left(39^{\circ} \mathrm{C}\right)$} \\
\hline & {$[\eta]$} & $k^{\prime}$ & $s_{0}{ }^{\circ}$ & $k_{s}$ & {$[\eta]_{\theta}$} & $\overline{k^{\prime}}$ & $S_{0}{ }^{\circ}$ & $k_{s}$ \\
\hline BB-8 & 0.203 & 0.38 & $3.35^{\mathrm{a}}$ & $0.260^{\mathrm{a}}$ & 0.142 & 0.56 & 4.00 & 0.10 \\
\hline BB-4 & 0.267 & 0.34 & $\ldots$ & $\ldots$ & 0.177 & 0.53 & 4.26 & 0.16 \\
\hline BH-1 & 0.811 & 0.34 & 5.95 & 0.816 & 0.395 & 0.59 & 10.25 & 0.28 \\
\hline BB-9 & 1.001 & 0.37 & 8.20 & 1.89 & 0.430 & 0.53 & 12.3 & 0.29 \\
\hline BB-6 & 1.154 & 0.33 & 9.66 & 2.53 & 0.528 & 0.58 & 13.0 & 0.44 \\
\hline BB-10 & 1.680 & 0.35 & $\ldots$ & $\ldots$ & 0.600 & 0.53 & 16.2 & 0.40 \\
\hline A-1 & 2.050 & 0.35 & 12.8 & 3.32 & 0.706 & 0.53 & 19.3 & 0.52 \\
\hline BB-5 & 2.933 & 0.34 & 15.2 & 4.67 & 0.910 & 0.53 & 23.9 & 0.79 \\
\hline BB-7 & 3.068 & 0.34 & 15.7 & 4.92 & 0.983 & 0.55 & 25.4 & 0.78 \\
\hline
\end{tabular}

Table II. Intrinsic viscosities and sedimentation coefficients of poly ( $\alpha$-methylstyrene) in benzene at $30^{\circ} \mathrm{C}$ and in cyclohexane at $39^{\circ} \mathrm{C}$

a Determined at rotor speed $59780 \mathrm{rpm}$. 
Dilute Solution Properties of Monodisperse Poly( $\alpha$-Methylstyrene)

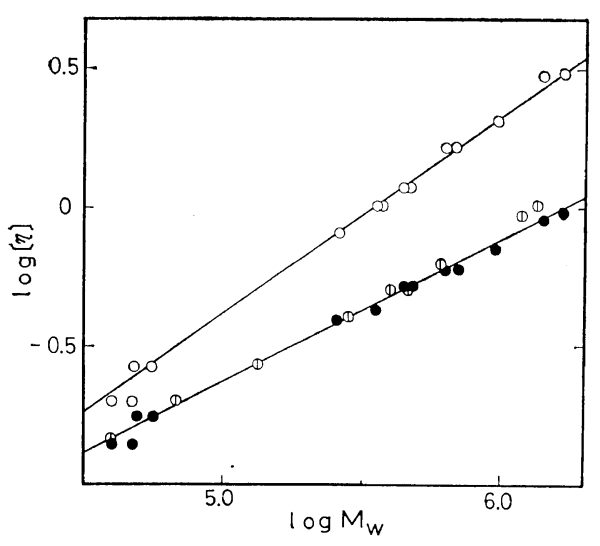

Figure 5. Double logarithmic plots of $[\eta]$ against $M_{w}$. Solvents: Benzene at $30^{\circ} \mathrm{C}$ (white circles); $\mathrm{Cy}-$ clohexane at $39^{\circ} \mathrm{C}$ (black circles, present data; circles with vertical bars, data by Noda et al.)

\section{DISCUSSION}

Excluded Volume Effect and the Draining Effect

The frictional coefficient $f$ of a polymer molecule at infinite dilution is related to the sedimentation coefficient $s_{0}{ }^{\circ}$ as

$$
s_{0}^{\circ}=M\left(1-\bar{v}^{\circ} \rho_{0}\right) / N_{A} f
$$

where $\bar{v}^{\circ}$ is the partial specific volume of the polymer at infinite dilution, and $\rho_{0}$ is the solvent density. According to the existing theories, ${ }^{6,19,20}$ this coefficient $f$ can be expressed in the form,

$$
f / \eta_{0}=6^{1 / 2} P\left\langle S^{2}\right\rangle^{1 / 2}
$$

where $\eta_{0}$ represents the solvent viscosity. In the original equation proposed by Mandelkern and Flory, ${ }^{21}$ the quantity $\boldsymbol{P}$ was a universal constant whose value was about 5.1-5.2. However, if either the excluded volume effect or the draining effect is introduced, the quantity $P$ becomes no longer constant and more or less dependent on the solvent nature and the molecular weight. A similar note is also applicable to Eq. 1 for the intrinsic viscosity which has been proposed originally by Flory and Fox. ${ }^{22}$ The quantity $\Phi$ is appreciably affected by either the excluded volume effect or the draining effect.

The effect of excluded volume on average dimensions of a polymer molecule is often expressed in terms of the linear expansion factor $\alpha_{s}$ which is defined by

$$
\left\langle S^{2}\right\rangle=\alpha_{s}{ }^{2}\left\langle S^{2}\right\rangle_{\theta}
$$

Here $\left\langle S^{2}\right\rangle_{\theta}$ represents the mean-square radius of gyration of the polymer immersed in theta solvent. Similarly, we can define the linear expansion factors $\alpha_{f}$ and $\alpha_{\eta}$ for the hydrodynamic effective radii of polymer coils. ${ }^{19}$ These are

$$
f=\alpha_{f} f_{\theta}
$$

and

$$
[\eta]=\alpha_{\eta}^{3}[\eta]_{\theta}
$$

respectively. Since $P$ and $\Phi$ in Eqs. 15 and 1 are not necessarily constant, these three expansion factors are not identical to one another: i.e.,

$$
\begin{gathered}
\alpha_{f}=\frac{P}{\boldsymbol{P}_{\theta}} \alpha_{s}, \quad \alpha_{\eta}=\left(\frac{\Phi}{\Phi_{\theta}}\right)^{1 / 3} \alpha_{s} \\
\text { and } \alpha_{\eta}=\frac{\beta}{\beta_{\theta}} \alpha_{f}
\end{gathered}
$$

where $\beta$ is the Scheraga-Mandelkern constant defined $b^{23}$

$$
\beta=\Phi^{1 / 3} P^{-1}=\frac{s_{0}{ }^{\circ}[\eta]^{1 / 3} \eta_{0} N_{A}}{\left(1-\bar{v}^{\circ} \rho_{0}\right) M^{2 / 3}}
$$

Figure 6 shows the test of Eq. 19, where the ratio $\alpha_{\eta}{ }^{3}=[\eta] /[\eta]_{\theta}$ is plotted against the ratio $\alpha_{f}=f / f_{\theta}$ using the double logarithmic scale. The present data are shown by open circles and they support the relationship

$$
\alpha_{\eta}=\alpha_{f}{ }^{\nu} \quad \text { with } \nu=1.00 \pm 0.07
$$

In the figure, the data obtained by Noda et al. for the same systems as the present are also shown by circles with bars for the sake of com-

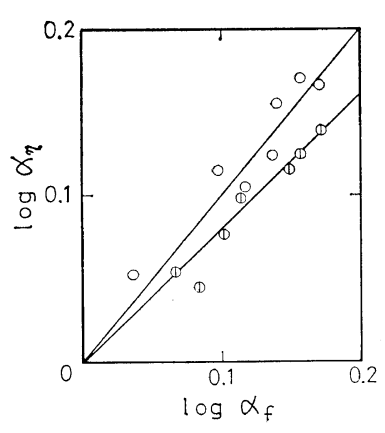

Figure 6. Relationship between hydrodynamic expansion factors $\alpha_{\eta}$ and $\alpha_{f}$. White circles, present data; circles with vertical bars, data by Noda et al. 
Table III. Various hydrodynamic functions for partially draining molecules ${ }^{\mathrm{a}}$

\begin{tabular}{|c|c|c|c|c|c|}
\hline \multirow{2}{*}{$h$} & \multicolumn{2}{|c|}{$\nu$} & \multirow{2}{*}{$\begin{array}{c}\Phi_{\theta} \times 10^{-21} \\
\mathbf{H} \\
\end{array}$} & \multirow{2}{*}{$\begin{array}{r}P_{\theta} \\
\mathrm{KR}\end{array}$} & \multirow{2}{*}{$\begin{array}{c}\beta_{\theta} \times 10^{-6} \\
\text { H,KR }\end{array}$} \\
\hline & KYH & SA & & & \\
\hline 0.1 & 5.02 & 3.48 & 0.155 & 1.08 & 4.97 \\
\hline 0.2 & 3.06 & 2.14 & 0.287 & 1.78 & 3.70 \\
\hline 0.5 & 1.92 & 1.36 & 0.592 & 2.92 & 2.87 \\
\hline 1.0 & 1.55 & 1.11 & 0.982 & 3.72 & 2.67 \\
\hline 2.0 & 1.39 & 0.99 & 1.42 & 4.31 & 2.61 \\
\hline 5.0 & 1.27 & 0.95 & 1.68 & 4.76 & 2.50 \\
\hline 10.0 & 1.25 & 0.91 & 2.27 & 4.93 & 2.67 \\
\hline 20.0 & 1.23 & 0.93 & 2.52 & 5.02 & 2.71 \\
\hline 50.0 & 1.23 & 0.93 & 2.69 & 5.08 & 2.74 \\
\hline 100.0 & 1.23 & 0.93 & 2.77 & 5.10 & 2.75 \\
\hline$\infty$ & 1.24 & 0.95 & $2.87^{b}$ & 5.12 & $2.77^{b}$ \\
\hline
\end{tabular}

a KYH: Kurata-Yamakawa, ${ }^{6}$ revised according to the Hearst theory. ${ }^{8}$ SA: Kurata-Yamakawa revised according to the Stockmayer-Albrecht theory. ${ }^{20} \mathrm{H}$ : Hearst. ${ }^{8}$ KR: Kirkwood-Riseman. ${ }^{5}$

b More recent estimates are given in Eqs. 24 and 25 .

parison. ${ }^{18}$ These data lead to the relationship, $\nu=0.8$.

According to theory, two values of $\nu$ are available for describing the dependence of $\alpha_{\eta}$ on $\alpha_{f}$ in the vicinity of theta temperature. These are 1.24 and 0.95 for the non-draining molecules, of which the former is obtained by Kurata and Yamakawa $^{6}$ and the latter by Stockmayer and Albrecht, ${ }^{20}$ respectively. For partial draining molecules, the index $\nu$ depends on the well-known hydrodynamic interaction parameter $h$ as shown in Table III. The parameter $h$ is defined by

$$
h=\zeta N^{1 / 2} /\left(12 \pi^{3}\right)^{1 / 2} a \eta_{0}
$$

where $N$ is the number of segments per molecule, $\zeta$ is the frictional coefficient of a segment and $a$ is the effective bond length. The difference in these two series of $\nu$ values arises from the approximations used in the evaluation of the statistical average of the inverse distance between chain elements, and the correct value is expected, from the nature of the used approximations, to lie between these two estimates. Our result is compatible with this conjecture.

Substitution of Eqs. 10 and 12 into Eq. 20 gives

$$
\beta=(2.43 \pm 0.03) \times 10^{6},
$$

which is in agreement with the previous estimate, $\beta_{\theta}=(2.41 \pm 0.07) \times 10^{6}$, within experimental error. On the other hand, the most recent theoretical estimates of $P_{\theta}$ and $\Phi_{\theta}$ are ${ }^{24,25}$

$$
P_{\sigma}=5.12 \text { and } \Phi_{\theta}=2.66 \times 10^{21}
$$

for the non-draining Gaussian coils, which yield

$$
\beta_{\theta}=2.70 \times 10^{6}
$$

Difference between these two values of $\beta$ is clearly beyond experimental error. It is, however, not clear whether this discrepancy shows evidence of the existence of a draining effect or not because the quantity $\beta$ is not very sensitive to the parameter $h$ and the above theoretical estimate of $\Phi_{\theta}$ is still approximate. Further investigations, especially those based on precise light scattering measurements, are needed to resolve the question.

Concentration Dependence of the Frictional Coefficient

In a preceding paper, we have demonstrated that the concentration dependence coefficient $k_{s}$ does not vanish at the theta point and is consistent with the prediction by Pyun and Fixman. ${ }^{26 .}$ Their expression for $k_{s}$ is written as

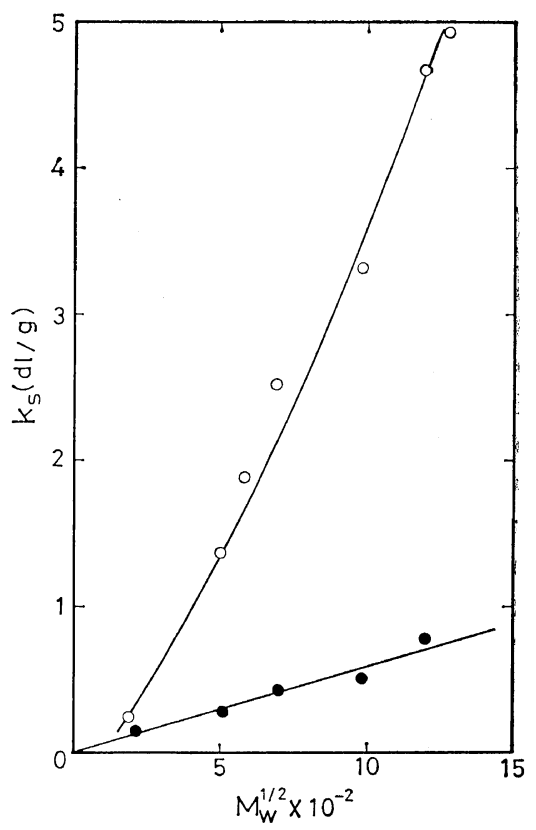

Figure 7. Plots of the concentration dependence coefficient $k_{s}$ against $M^{1 / 2}$. Solvents: Benzene at $30^{\circ} \mathrm{C}$ (white circles); Cyclohexane at $39^{\circ} \mathrm{C}$ (black circles). 


$$
k_{s}=[7.16-\kappa(A)] \frac{4}{300}\left(\frac{M}{N_{A} \pi}\right)^{2}\left[\frac{\left(1-\bar{v}^{\circ} \rho_{0}\right)}{6 \eta_{0} s_{0}^{\circ}}\right]^{3}
$$

where $A$ is a parameter representing the thermodynamic interaction between chain elements. For large values of $\boldsymbol{A}$ which correspond to good solvent systems, the function $\kappa(A)$ can be approximated by the asymptotic formula

$$
\kappa(A)=6.39 /(A+2.6)^{1 / 2} .
$$

In Figure 7, we show by open circles our data of $k_{s}$ which were obtained in benzene at $30^{\circ} \mathrm{C}$. These data well coincide with the theoretical curve which is calculated by Eqs. 26 and 27 with $\kappa(A)=0.63$ or $A=100$. Our theta solvent data indicated by closed circles are also shown in the figure. ${ }^{10}$

We have performed sedimentation velocity measurements also below the theta temperature in cyclohexane. It has been found that the concentration dependence of $s_{0}{ }^{\circ}$ vanishes at about $27^{\circ} \mathrm{C}$, though the data are not reproduced here.

\section{Unperturbed Dimension}

Figure 8 shows the familiar viscosity plot, where the values of $[\eta] / M^{1 / 2}$ are plotted against $M^{1 / 2}$. As was pointed out by Kurata, Stockmayer and Fixman, ${ }^{19,27}$ two series of data, one in benzene and the other in cyclohexane, have a common intercept in this plot, showing the unperturbed

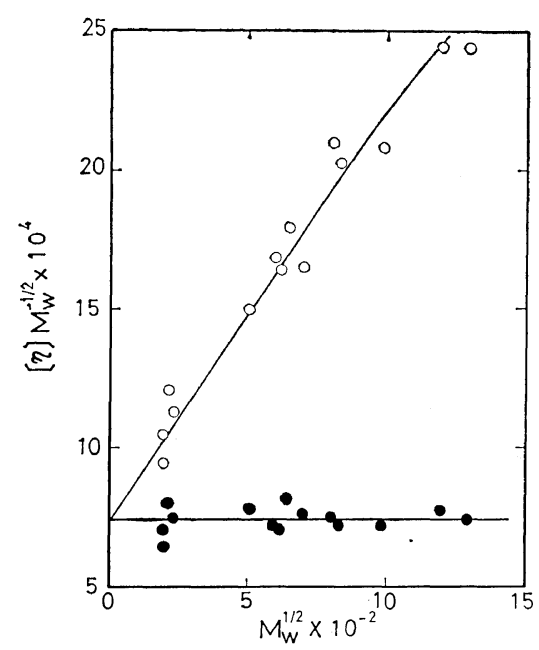

Figure 8. Plots of $[\eta] M^{-1 / 2}$ against $M^{1 / 2}$. Solvents: Benzene at $30^{\circ} \mathrm{C}$ (white circles); Cyclohexane at $39^{\circ} \mathrm{C}$ (black circles). dimension of this polymer to be essentially independent of the solvents used. From the intercept, we obtain

$$
\left\{\left\langle R^{2}\right\rangle_{\theta} / M\right\}^{1 / 2}=6.50 \times 10^{-9} \mathrm{~cm}
$$

where $R$ is the end-to-end distance of a polymer chain. This estimate of the unperturbed dimension is in satisfactory agreement with that by Noda et al. ${ }^{18}$ and by Cowie et al. ${ }^{28}$

Acknowledgement. One of the authors (KS) wishes to express a debt to the late Dr. Masaaki Fukatsu for his continued guidance and stimulating discussions. We are indebted to Dr. Hiroyasu Utiyama for many helpful discussions. The technical assistance of Mr. Takashi Kageyama in ultracentrifuge experiments is also acknowledged.

\section{REFERENCES}

1. G. C. Berry, J. Chem. Phys., 46, 1338 (1967).

2. H. Inagaki, H. Suzuki, and M. Kurata, J. Polym. Sci., 15, 409 (1966).

3. K. Kawahara, T. Norisuye, and H. Fujita, $J$. Chem. Phys., 49, 4339 (1968).

4. G. Tanaka, S. Imai, and H. Yamakawa, unpublished.

5. J. G. Kirkwood and J. Riseman, J. Chem. Phys., 16, 565 (1948).

6. M. Kurata and H. Yamakawa, J. Chem. Phys., 29, 311 (1958).

7. B. H. Zimm, J. Chem. Phys., 24, 269 (1956).

8. J. E. Hearst, J. Chem. Phys., 37, 2547 (1952).

9. M. Fixman, J. Chem. Phys., 45, 785, 793 (1966).

10. M. Abe, K. Sakato, T. Kageyama, M. Fukatsu, and M. Kurata, Bull. Chem. Soc. Japan, 41, 2330 (1968).

11. C. I. Carr and B. H. Zimm, J. Chem. Phys., 18, 1616 (1950).

12. A. Kruis, Z. Phys. Chem., 348, 13 (1936).

13. H. Utiyama, N. Tagata, and M. Kurata, J. Phys. Chem., 73, 1448 (1969).

14. P. E. Hexner, L. E. Radford, and J. W. Beams, Proc. Natl. Acad. Sci., U. S., 47, 1848 (1961).

15. G. C. Berry, J. Chem. Phys., 44, 4550 (1966).

16. H. Utiyama, N. Sugi, M. Kurata, and M. Tamura, Bull. Inst. Chem. Res. Kyoto Univ., 46, 77, 198 (1968).

17. H. Fujita, Mathematical Theory of Sedimentation Analysis (Academic Press Inc., New York, 1962).

18. I. Noda, S. Saito, T. Fujimoto, and M. Naga- 


\section{K. Sakato and M. Kurata}

sawa, J. Phys. Chem., 71, 4048 (1967).

19. M. Kurata and W.H. Stockmayer, Fortsh. Hochpolym. Forsch., 3, 196 (1963).

20. W. H. Stockmayer and A. C. Albrecht, J. Polym. Sci., 32, 215 (1958).

21. L. Mandelkern and P. J. Flory, J. Chem. Phys., 20, 212 (1952).

22. P. J. Flory and T. G Fox, Jr., J. Amer. Chem. Soc., 73, 1904 (1951).

23. H. A. Scheraga and L. Mandelkern, J. Amer. Chem. Soc., 75, 179 (1953).

24. J. G. Kirkwood, J. Polym. Sci., 12, 1 (1954).
25. C. W. Pyun and M. Fixman, J. Chem. Phys., 42, 3838 (1965); 44, 2107 (1966).

26. C. M. Pyun and M. Fixman, J. Chem. Phys., 41, 937 (1964).

27. W. H. Stockmayer and M. Fixman, in First Biannual American Chemical Society Polymer Symposium, 1963 (J. Polym. Sci., Part C, 1), H. W. Starkweather, Jr., Ed., Interscience, New York, 1963, p. 137.

28. J. W. G. Cowie, S. Bywater, and D. J. Worsfold, Polymer, 8, 105 (1967). 